\title{
INDECOMPOSABLE HOMOGENEOUS PLANE CONTINUA ARE HEREDITARILY INDECOMPOSABLE
}

\author{
BY
}

\author{
CHARLES L. HAGOPIAN
}

\begin{abstract}
F. Burton Jones [7] proved that every decomposable homogeneous plane continuum is either a simple closed curve or a circle of homogeneous nonseparating plane continua. Recently the author [5] showed that no subcontinuum of an indecomposable homogeneous plane continuum is hereditarily decomposable. It follows from these results that every homogeneous plane continuum that has a hereditarily decomposable subcontinuum is a simple closed curve. In this paper we prove that no subcontinuum of an indecomposable homogeneous plane continuum is decomposable. Consequently every homogeneous nonseparating plane continuum is hereditarily indecomposable. Parts of our proof follow one of R. H. Bing's arguments [2]. At the Auburn Topology Conference in 1969, Professor Jones [8] outlined an argument for this theorem and stated that the details would be supplied later. However, those details have not appeared.
\end{abstract}

1. Definitions. A space is homogeneous if for each pair $p, q$ of its points there exists a homeomorphism of the space onto itself that takes $p$ to $q$.

A continuum is a nondegenerate compact connected metric space. A continuum is of type $A^{\prime}$ provided it is irreducible between a pair of its points and admits a monotone upper semicontinuous decomposition, each of whose elements has void interior, whose quotient space is an arc [14].

A finite collection $\left\{L_{i}: 1 \leqslant i \leqslant n\right\}$ of open sets is a chain provided that $L_{i} \cap L_{j} \neq \varnothing$ if and only if $|i-j| \leqslant 1$.

Throughout this paper $R^{2}$ is the Cartesian plane with metric $\rho$. The closed interval with end points $p$ and $q$ in $R^{2}$ is denoted by $\langle p, q\rangle$. We shall denote the closure and the boundary of a given set $H$ by $\mathrm{Cl} H$ and $\mathrm{Bd} H$ respectively. The union of a collection $H$ of sets is denoted by St H. For definitions of unfamiliar terms and phrases see [5], [9], and [13].

Received by the editors October 7, 1975.

AMS (MOS) subject classifications (1970). Primary 54F15, 54F20, 54F65, 57A05; Secondary 54F50, 54G15, 54H15, 57A60.

Key words and phrases. Homogeneity, indecomposable continua, nonseparating plane continua, hereditarily indecomposable continua, upper semicontinuous decomposition, pseudoarc. 
2. Preliminaries. In this section $M$ is an indecomposable homogeneous continuum in $R^{2}$ and $E$ is a type $A^{\prime}$ subcontinuum of $M$. Let $k: E \rightarrow[0,1]$ be the quotient map associated with the minimal admissible decomposition of $E$ [5, Lemma 3]. Hereafter, we shall refer to this decomposition as simply the decomposition of $E$.

Let $G=\left\{G_{i}: 1 \leqslant i \leqslant n\right\}$ be a chain in $R^{2}$ that covers $E$.

The chain $G$ is said to follow $E$ provided that

(1) $k^{-1}(0) \subset G_{1}$ and $k^{-1}(1) \subset G_{n}$,

(2) each element of the decomposition of $E$ lies in an element of $G$, and

(3) if $0 \leqslant r<s \leqslant 1$ and $k^{-1}(r) \cup k^{-1}(s)$ is contained in an element $G_{i}$ of $G$, then the union of all elements of $G$ that intersect $G_{i}$ contains $k^{-1}[[r, s]]$.

The chain $G$ is said to be free relative to $M$ if $M \cap \mathrm{Bd}(\mathrm{St} G)$ is contained in $M \cap \mathrm{Bd}\left(G_{1} \cup G_{n}\right)$.

A chain $\left\{L_{i}: 1 \leqslant i \leqslant n\right\}$ in $R^{2}$ is called an ordered refinement of $G$ if for each $i$, the link $L_{i}$ is contained in $G_{i}$.

We call $X=k^{-1}(0)$ and $Z=k^{-1}(1)$ the end sets of $E$. No proper subcontinuum of $E$ intersects both $X$ and $Z$ [14, Theorem 8, p. 14]. A subcontinuum $F$ of $M$ is called an extension of $E$ away from $X$ if $F$ is a continuum of type $A^{\prime}$ that contains $E$ and has $X$ as an end set. We define $R(X, E)$ to be the union of all extensions of $E$ away from $X$. We let $R(E)$ denote $R(X, E) \cup R(Z, E)$.

LEMMA 1. The continuum $M$ is atriodic and hereditarily unicoherent [5, Lemma 1].

Let $\epsilon$ be a positive number. A homeomorphism $h$ of $M$ onto $M$ is called an $\epsilon$-homeomorphism if $\rho(v, h(v))<\epsilon$ for each point $v$ of $M$. Our next lemma follows from a theorem of E. G. Effros [3, Theorem 2.1] involving topological transformation groups.

LEMMA 2. Suppose $\epsilon$ is a given positive number and $x$ is a point of $M$. Then $\{y \in M$ : an $\epsilon$-homeomorphism of $M$ onto $M$ takes $x$ to $y\}$ is an open subset of $M$ that contains $x[5$, Lemma 4 (proof)].

LEMMA 3. Let $Y$ be an element of the decomposition of $E$ distinct from the end sets $X$ and $Z$ of $E$. Let $F$ be a type $A^{\prime}$ subcontinuum of $M$ with end sets $T$ and $V$, and let $U$ be an element of the decomposition of $F$ distinct from $T$ and $V$. Suppose $h$ is a homeomorphism of $M$ onto $M$ such that $U \cap h[Y] \neq$ $\varnothing$ and $U \cap h[X \cup Z]=\varnothing=h[Y] \cap(T \cup V)$. Then $h[Y]=U$.

Proof. This lemma follows from the argument presented in paragraphs 9 through 11 in the proof of Theorem 1 of [5].

LEMMA 4. The collection $F$ of extensions of $E$ away from the end set $X$ is linearly ordered by inclusion and does not have a maximal element. 
Proof. Let $F$ be an element of $F$, and let $d: F \rightarrow[0,1]$ be the quotient map associated with the decomposition of $F$. We assume $X=d^{-1}(0)$. There exists a positive number $s$ such that $d[E]=[0, s]$. Since $F$ is irreducible, for each positive number $r$ less than $s$, the set $d^{-1}(r)$ is in $E$. Since the decompositions of $E$ and $F$ are minimal, each element of the decomposition of $E$ (except possibly $\left.k^{-1}(1)\right)$ is an element of the decomposition of $F$.

If $H$ is an element of $F$, then $F \subset H$ or $H \subset F$; for otherwise, the continuum $H \cap d^{-1}[[s, 1]]$ (Lemma 1) would separate $F \cup H$ into three nonempty disjoint open sets, which contradicts the fact that $M$ is atriodic. It follows that $F$ is linearly ordered by inclusion.

To see that no element of $F$ is maximal, let $p$ be a point of the end set $d^{-1}(1)$ of $F$. Define $\epsilon$ to be $\rho\left(X, d^{-1}[[1 / 2,1]]\right)$. By Lemma 2, a point $q$ of $d^{-1}[[1 / 2,1)]$ and an $\epsilon$-homeomorphism $h$ of $M$ onto $M$ exist such that $h(p)=$ $q$. Let $U$ be the element of the decomposition of $F$ that contains $q$. Note that $X \cap h^{-1}[U]=\varnothing=d^{-1}(1) \cap h^{-1}[X]$. Since $F$ is irreducible between $X$ and $d^{-1}(1)$, it is a proper subset of an element of $F$ that is contained in $F \cup h^{-1}[F]$ (Lemma 1). This implies that $F$ does not have a maximal element.

LEMMA 5. The decomposition of $E$ is continuous.

Proof. Let $A$ denote the decomposition of $E$. Let $\left\{y_{i}\right\}$ and $\left\{Y_{i}\right\}$ be sequences such that each $Y_{i}$ is an element of $A$ and each $y_{i}$ is a point of $Y_{i}$. Assume $\left\{y_{i}\right\}$ converges to a point $y$ belonging to an element $Y$ of $A$. Define $L$ to be the limit superior of $\left\{Y_{i}\right\}$. Since $A$ is upper semicontinuous, $Y$ contains $L$.

We must show that $Y=L$. To accomplish this we assume the contrary. Let $p$ be a point of $Y-L$. There exists a type $A^{\prime}$ continuum $F$ in $R(E)$ that is the union of two extensions of $E$ such that the end sets $T$ and $V$ of $F$ miss $E$ (Lemma 4). Let $B$ denote the decomposition of $F$. Each element of $A$ is contained in an element of $B$. In fact, each element of $A$ that is not an end set of $E$ is an element of $B$. Let $U$ be the element of $B$ that contains $Y$. Define $\epsilon$ to be the minimum of $\rho(p, L)$ and $\rho(U, T \cup V)$.

By Lemma 2, an integer $i$ and an $\epsilon / 2$-homeomorphism $h$ of $M$ onto $M$ exist such that (1) $h(y)=y_{i}$, (2) $Y_{i} \in B$, and (3) $\rho\left(Y_{i},\{p\} \cup T \cup V>\epsilon / 2\right.$. It follows from Lemma 3 that $h[U]=Y_{i}$. Since $\rho\left(p, Y_{i}\right)>\epsilon / 2$ and $h$ is an $\epsilon / 2$-homeomorphism, this is a contradiction. Hence $Y=L$ and, therefore, $A$ is continuous.

LEMMA 6. The continuum $\mathrm{Cl} R(E)$ is indecomposable.

Proof. Let $X$ and $Z$ be the end sets of $E$. Note that if $F$ is an element 
of the set $F$ of extensions of $E$ away from $X$, then the decomposition of $F$ is a continuous collection (Lemma 5 ) that has the decomposition of $E$ as a subcollection.

First we prove that $\mathrm{Cl} R(X, E)$ is indecomposable. To accomplish this we assume the contrary. Suppose there exist proper subcontinua $B$ and $C$ of $\mathrm{Cl} R(X, E)$ such that $\mathrm{Cl} R(X, E)=B \cup C$.

Assume without loss of generality that $X$ intersects $B$. Since $E$ is irreducible and $M$ is hereditarily unicoherent, and since $X$ does not have interior relative to $E$, it follows that $B$ contains $X$.

The sets $X$ and $C$ are disjoint. For suppose on the contrary that $X$ intersects $C$. Then $C$ contains $X$. Let $H$ be an element of $F$ such that $H \cap(B-C) \neq$ $\varnothing \neq H \cap(C-B)$. Since $B \cap H$ and $C \cap H$ are continua, this violates the irreducibility of $H$.

Define $\epsilon$ to be $\rho(X, C)$.

Let $K$ be an element of $F$ that meets $C-B$. Note that $B-C$ is contained in $K$.

Since $M$ is second countable, there exists a monotone strictly increasing sequence $\left\{F_{i}\right\}$ of elements of $F$ such that $R(X, E)=\bigcup_{i=1}^{\infty} F_{i}$ (Lemma 4). For each $i$, let $z_{i}$ be a point of the end set of $F_{i}$ opposite $X$. Define $c$ to be a point of $\mathrm{Cl} R(X, E)$ that is a limit point of $\left\{z_{i}\right\}$. Note that $c$ belongs to $C$.

The point $c$ belongs to $\mathrm{Cl} R(X, E)-R(X, E)$. To see this assume $c$ is a point of $R(X, E)$. Let $L$ be an element of $F$ that contains $K \cup\{c\}$, and let $w$ be a point of $R(X, E)-L$. Define $\delta$ to be the minimum of $\epsilon$ and $\rho(w, L)$. It follows from Lemma 2 that an integer $i$ and a $\delta$-homeomorphism $f$ of $M$ onto $M$ exist such that $f(c)=z_{i}$ and $w$ belongs to $F_{i}$. Since $M$ is hereditarily unicoherent and $f[L]$ contains $z_{i}$ and misses $w$, and since $F_{i}$ is irreducible, the sets $L$ and $f[L]$ are disjoint. Since $M$ is atriodic, it follows that $f[L]$ is a subset of $R(X, E)$. However, $B-C \subset K \subset L$ and $f[X] \subset M-C$. This implies that $f[L]$ is not contained in $B \cup C$, which contradicts the assumption that $\mathrm{Cl} R(X, E)=B \cup C$.

Let $\epsilon_{1}$ be a positive number less than $\epsilon$ such that if $Y$ is an element of the decomposition of $K$, then $\left\{v \in M: \rho(v, Y)<\epsilon_{1}\right\}$ does not contain $K$. By Lemma 2, there exists an $\epsilon_{1}$-homeomorphism $h_{1}$ of $M$ onto $M$ such that $h_{1}(c)$ belongs to $R(X, E)$. Let $N$ be an element of $F$ that contains $K \cup\left\{h_{1}(c)\right\}$.

The sets $N$ and $h_{1}^{-1}[N]$ are disjoint. For suppose on the contrary that $N \cap h_{1}^{-1}[N] \neq \varnothing$. Then since no element $Y$ of the decomposition of $N$ has the property that $h_{1}^{-1}[Y]$ contains $N$, the point $c$ belongs to $R(E)$. Since $c$ does not belong to $R(X, E)$, there exists an extension of $E$ away from $Z$ that contains $c$. Therefore $R(E)$ contains a continuum whose intersection with $C$ is not connected, which is impossible (Lemma 1). 
Let $\epsilon_{2}$ be the minimum of $\epsilon_{1}$ and $\rho\left(N, h_{1}^{-1}[N]\right)$. Let $h_{2}$ be an $\epsilon_{2}$ homeomorphism of $M$ onto $M$ such that $h_{2}(c)$ is in $R(X, E)$ (Lemma 2). Define $P$ to be an element of $F$ that contains $N \cup\left\{h_{2}(c)\right\}$. By the preceding argument, $P$ and $h_{2}^{-1}[P]$ are disjoint. Hence $N, h_{1}^{-1}[N]$, and $h_{2}^{-1}[N]$ are mutually disjoint sets that meet $M-C$. Thus $C \cup N \cup h_{1}^{-1}[N] \cup h_{2}^{-1}[P]$ is a triod, and this is a contradiction of Lemma 1. It follows that $\mathrm{Cl} R(X, E)$ is indecomposable.

We complete this proof by showing that $\mathrm{Cl} R(X, E)=\mathrm{C} 1 R(E)$. To accomplish this assume there exists an extension $Q$ of $E$ away from $Z$ that is not contained in $\mathrm{Cl} R(X, E)$. By Lemma 2, there exist homeomorphisms $f_{1}$ and $f_{2}$ of $M$ onto $M$ near the identity such that $Q \cup f_{1}[Q] \cup f_{2}[Q] \cup \mathrm{Cl} R(X, E)$ is a triod (see the second paragraph in the proof of Theorem 1 of [5]). It follows from this contradiction that $\mathrm{Cl} R(X, E)$ contains $R(Z, E)$. Hence $\mathrm{Cl} R(X, E)=$ $\mathrm{Cl} R(E)$.

Since $M$ is atriodic, $R(E)$ is a composant of $\mathrm{Cl} R(E)$. A continuum in $R(E)$ that intersects more than one element of the decomposition of some extension of $E$ is said to be essential to $R(E)$. Note that every continuum essential to $R(E)$ is of type $A^{\prime}$ (Lemma 5).

LEMMA 7. For each positive number $\epsilon$, there exists a positive number $\delta$ such that if $h$ is a $\delta$-homeomorphism of $M$ onto $M$, then $h[R(E)]$ has the following property. If $H$ is a continuum essential to $h[R(E)]$ with end sets $T$ and $V$ such that $\rho(T, V)<\delta$ and if there exists an element $U$ of the decomposition of $H$ such that $\rho(T, U)>\epsilon$, then each point of $R(E)$ is within $\epsilon$ of $H$.

Proof. Assume the contrary. Let $\epsilon$ be a positive number such that for each positive integer $i$, there exists an $i^{-1}$-homeomorphism $h_{i}$ of $M$ onto $M$ with the following properties. A point $p_{i}$ of $R(E)$ and a continuum $H_{i}$ essential to $h_{i}[R(E)]$ having end sets $T_{i}$ and $V_{i}$ exist such that $\rho\left(p_{i}, H_{i}\right)>\epsilon$ and $\rho\left(T_{i}, V_{i}\right)<$ $i^{-1}$. Furthermore, there exists an element $U_{i}$ of the decomposition of $H_{i}$ such that $\rho\left(T_{i}, U_{i}\right)>\epsilon$.

For each $i$, let $u_{i}$ be a point of $U_{i}$, and let $t_{i}$ and $v_{i}$ be points of $T_{i}$ and $V_{i}$, respectively, such that $\rho\left(t_{i}, v_{i}\right)<i^{-1}$. Let $t, u$, and $p$ be points of $\mathrm{Cl} R(E)$ that are limit points of $\left\{t_{i}\right\},\left\{u_{i}\right\}$, and $\left\{p_{i}\right\}$, respectively. Let $A=\{x \in M$ : $\rho(x, p)<\epsilon / 4\}$ and $B=\{x \in M: \rho(x, p)<\epsilon / 2\}$. Assume without loss of generality that $\left\{t_{i}\right\},\left\{u_{i}\right\}$, and $\left\{p_{i}\right\}$ converge to $t, u$, and $p$, respectively, and each $H_{i}$ misses $B$.

By Lemma 2, an integer $i$ and a homeomorphism $f$ of $M$ onto $M$ exist such that a continuum $F$ essential to $f h_{i}[R(E)]$ contains $u$, misses $t$, and intersects $A$. Define $\epsilon^{\prime}$ to be the minimum of $\epsilon / 4$ and $\rho(t, F)$. It follows from Lemma 2 that an integer $j$ and an $\epsilon^{\prime}$-homeomorphism $g$ of $M$ onto $M$ exist such that $g(u)=u_{j}$ 
and $\left\{t_{j}, v_{j}\right\} \cap g[F]=\varnothing$. Note that $g[F] \cup H_{j}$ is a triod in $M$. This contradiction completes our proof.

LEMMA 8. Every chain in $R^{2}$ that follows $E$ has an ordered refinement that follows $E$ and is free relative to $M$.

Proof. Let $G=\left\{G_{i}: 1 \leqslant i \leqslant n\right\}$ be a chain in $R^{2}$ that follows $E$. Since $M$ is atriodic, there exist open sets $A$ and $B$ in $G_{1}$ and $G_{n}$, respectively, such that $E$ is a component of $M-(A \cup B)$.

Since no component of $M-(A \cup B)$ meets both $E$ and $M-$ St $G$, there exist disjoint open sets $C$ and $D$ in $R^{2}$ such that (1) $C \supset E$, (2) $D \supset M-$ St $G$, and (3) $C \cup D \supset M-(A \cup B)$ [13, Theorem 44, p. 15].

Let $L_{1}=G_{1}$ and $L_{n}=G_{n}$. For each integer $i(1<i<n)$, define $L_{i}$ to be $C \cap G_{i}$. The chain $\left\{L_{i}: 1 \leqslant i \leqslant n\right\}$ is an ordered refinement of $G$ that follows $E$ and is free relative to $M$.

Let $S$ be the equivalence relation on $R^{2}$ that relates distinct points $p$ and $q$ if and only if $p$ and $q$ belong to an element of the decomposition of $E$. Since $M$ is indecomposable and homogeneous, no proper subcontinuum of $M$ separates $R^{2}$ [5, Lemma 1 (second paragraph of proof)]. Hence we can assume that the quotient space $R^{2} / S$ is $R^{2}$ [12]. Let $\pi$ be the quotient map associated with $S$.

Distinct elements $U$ and $Y$ of the decomposition of $E$ that are not end sets are said to be decomposably accessible from a side of $E$ if there exist arc segments $A$ and $B$ in $R^{2}-\pi[M]$ such that (1) $\pi[U] \in \mathrm{Cl} A,(2) \pi[Y] \in \mathrm{Cl} B$, and (3) $\mathrm{Cl} A$ and $\mathrm{Cl} B$ abut on the same side of the $\operatorname{arc} \pi[E]$ in $R^{2}[2$, p. 215].

LEMMA 9. Let $U$ and $Y$ be elements of the decomposition of $E$ that are not end sets. If $Y$ is accessible from $R^{2}-M$, then $U$ and $Y$ are decomposably accessible from a side of $E$.

Proof. Assume without loss of generality that $U=k^{-1}(1 / 3)$ and $Y=$ $k^{-1}(1 / 2)$ ( $k$ is the quotient map associated with the decomposition of $E$ ). Let $I$ be an arc in $R^{2}$ and $y$ be a point of $Y$ such that $I \cap M=\{y\}$.

Let $C=k^{-1}[[0,1 / 2]], D=k^{-1}[[1 / 3,1]], H_{1}=k^{-1}[[0,1 / 4]], H_{2}=$ $k^{-1}[[1 / 4,3 / 4]]$, and $H_{3}=k^{-1}[[3 / 4,1]]$.

Letting $x$ be a point of $I-\{y\}$, we define $\epsilon$ to be one-third the minimum of $\rho(x, E), \rho\left(I, H_{1} \cup H_{3}\right), \rho\left(C, H_{3}\right), \rho\left(D, H_{1}\right)$, and $\rho\left(H_{2}, k^{-1}(0) \cup k^{-1}(1)\right)$.

Let $G=\left\{G_{1}, G_{2}, G_{3}\right\}$ be a chain in $R^{2}$ that follows $E$ such that (1) for $i=1,2$, and $3, H_{i} \subset G_{i}$, each point of $G_{i}$ is within $\epsilon$ of $H_{i}$, and $\pi\left[G_{i}\right]$ is an open disk, and (2) for $i=1$ and $2, \pi\left[G_{i}\right] \cap \pi\left[G_{i+1}\right]$ is an open disk. Note that $I$ intersects $\mathrm{Bd} G_{2}$ and misses $\mathrm{Cl}\left(G_{1} \cup G_{3}\right)$.

Let $L=\left\{L_{1}, L_{2}, L_{3}\right\}$ be an ordered refinement of $G$ that follows $E$ and is free relative to $M$ (Lemma 8 ). 
We assume without loss of generality that $\pi[E]$ is lying on a horizontal line $B$ in $R^{2}$ and $\pi[I-\{y\}]$ is below $B$ on a vertical line.

Assume $U$ and $Y$ are not decomposably accessible from a side of $E$. Let $A$ be a vertical line segment in $R^{2}$ below $B$ that has $\pi[U]$ as an end point. It follows from Lemma 2 that a point $u$ of $U$ and an $\epsilon$-homeomorphism $h$ of $M$ onto $M$ exist such that (1) $h(u) \in L_{2} \cap(M-E),(2) \pi h(u) \in A$, and (3) the segment in $A$ from $\pi(u)$ to $\pi h(u)$ is in $\pi\left[L_{2}\right]$.

Since $M$ is atriodic and $h(u)$ is not in $E$, the sets $G_{2} \cap E$ and $G_{2} \cap h[E]$ are disjoint. Note that $\pi h[D]$ contains $\pi h(u)$, intersects $\pi\left[G_{3}\right]$, and misses $\pi\left[C \cup I \cup C G_{1}\right]$. Since $L$ is free relative to $M$ and $\pi\left[C \cup \eta \cap \pi\left[C G_{3}\right]=\right.$ $\varnothing$, this is impossible [13, Theorem 28, p. 156]. It follows that $U$ and $Y$ are decomposably accessible from a side of $E$.

3. Principal results.

THEOREM 1. If $M$ is an indecomposable homogeneous continuum in $R^{2}$, then $M$ is hereditarily indecomposable.

Proof. Assume $M$ has a decomposable subcontinuum. It follows from [5, Theorem 1 (paragraphs 1,2 , and 3 of proof)] that $M$ has a subcontinuum $E$ of type $A^{\prime}$. Let $k: E \rightarrow[0,1]$ be the quotient map associated with the decomposition of $E$.

Since $M$ is homogeneous, we can assume without loss of generality that $k^{-1}[(0,1)]$ is accessible from $R^{2}-M$.

Define $S$ to be the equivalence relation on $R^{2}$ that relates distinct points $p$ and $q$ if and only if $\{p, q\}$ is a subset of an element of the decomposition of $E$. Let $\pi$ be the quotient map associated with $S$.

Assume without loss of generality that $R^{2} / S=R^{2}$, that the arc $\pi[E]$ is lying on the horizontal axis of $R^{2}$, that $\pi\left[k^{-1}(i / 22)\right]=(i, 0)$ for each integer $i$ $(0 \leqslant i \leqslant 22)$, and that each point of $\pi[E]$ is an end point of an arc segment in $R^{2}-\pi[M]$ below $\pi[E]$ (Lemma 9).

For each $i(1 \leqslant i \leqslant 5)$, let $o_{i}=(4 i-1,-1), p_{i}=(4 i-1,0)$, and $q_{i}=$ $(4 i-1,1)$ in $R^{2}$, and let

$$
\left.A_{i}=\pi^{-1}\left[\wp_{i}, q_{i}\right\rangle\right] \text { and } B_{i}=k^{-1}[[0,(2 i-1) / 11] \cup[2 i / 11,1]] \text {. }
$$

Define $\epsilon_{1}$ to be one-third the minimum of the union of

$$
\begin{aligned}
& \left\{\rho\left(A_{i}, B_{i}\right): 1 \leqslant i \leqslant 5\right\}, \quad\left\{\rho\left(E, \pi^{-1}\left(q_{i}\right)\right): 1 \leqslant i \leqslant 5\right\}, \\
& \left\{\rho\left(k^{-1}(0) \cup k^{-1}(1), k^{-1}[[1 / 11,10 / 11]]\right)\right\}, \text { and } \\
& \left\{\rho\left(k^{-1}[[0, i / 11]], k^{-1}[[(i+1) / 11,1]]\right): 1 \leqslant i \leqslant 9\right\} .
\end{aligned}
$$


Note that if $h$ is an $\epsilon_{1}$-homeomorphism of $M$ onto $M$, then the chain formed by taking $\epsilon_{1}$-neighborhoods in $R^{2}$ of the elements of $\left\{k^{-1}[[(i-1) / 11, i / 11]]: 1 \leqslant i \leqslant 11\right\}$ follows $h[E]$.

For each point $x$ of $E$, let $W_{x}$ be the open set $\left\{y \in M\right.$ : an $\epsilon_{1}$ homeomorphism of $M$ onto $M$ takes $x$ to $y$ \} (Lemma 2), and let $G_{x}$ be an open set in $\left\{z \in R^{2}: \rho(x, z)<\epsilon_{1}\right\}$ such that $W_{x}=M \cap G_{x}$.

For each $i(1 \leqslant i \leqslant 11)$, let $G_{i}=\bigcup\left\{G_{x}: x \in k^{-1}[[(i-1) / 11, i / 11]]\right\}$. Using the method described in the proof of Lemma 8 , we define an ordered refinement $L=\left\{L_{i}: 1 \leqslant i \leqslant 11\right\}$ of the chain $\left\{G_{i}: 1 \leqslant i \leqslant 11\right\}$ that follows $E$ and is free relative to $M$ having the following property:

PROPERTY 1. For each point $y$ of $M \cap \bigcup\left\{L_{i}: 2 \leqslant i \leqslant 10\right\}$, there exists an $\epsilon_{1}$-homeomorphism $h$ of $M$ onto $M$ such that $y$ belongs to $h[E]$ and $L$ follows $h[E]$. (Here the open sets $A$ and $B$ in the proof of Lemma 8 are in $G_{1}-G_{2}$ and $G_{11}-G_{10}$ respectively.)

It follows from the proof of Lemma 9 that there exists a horizontal interval $I_{1}$ below $\left\langle p_{1}, p_{5}\right\rangle$ in $R^{2}-\pi[M]$ such that the end points of $I_{1}$ are in $\left\langle o_{1}, p_{1}\right\rangle \cup$ $\left\langle o_{5}, p_{5}\right\rangle$ and no point of $\pi[M]$ lies between $I_{1}$ and $\left\langle p_{1}, p_{5}\right\rangle$.

Let $I_{2}$ be an arc above $\left\langle p_{1}, p_{5}\right\rangle$ in $\pi[(\mathrm{St} L)-M]$ that is irreducible between $\left\langle p_{1}, q_{1}\right\rangle$ and $\left\langle p_{5}, q_{5}\right\rangle$ such that every point of $R^{2}$ between $I_{2}$ and $\left\langle p_{1}, p_{5}\right\rangle$ is in $\pi\left[\right.$ St L]. We find that there exists such an $\operatorname{arc} I_{2}$ as follows. Let $y$ be a point of $M \cap L_{6}$ that is accessible from $R^{2}-M$ such that $\pi(y)$ is above $\pi[E]$. Let $h$ be an $\epsilon_{1}$-homeomorphism of $M$ onto $M$ such that $h[E]$ contains $y$ and is followed by $L$ (Property 1). There exists an arc $A$ in (St $L)-M$ to one side of $h[E]$ that meets $L_{1}$ and $L_{11}$ (Lemma 9 (proof)). By choosing $y$ sufficiently close to $E$ and $h$ sufficiently close to the identity, we get that every point of $R^{2}$ between $\left\langle p_{1}, p_{5}\right\rangle$ and a subarc of $\pi[A]$ irreducible between $\left\langle p_{1}, q_{1}\right\rangle$ and $\left\langle p_{5}, q_{5}\right\rangle$ belongs to $\pi[$ St $L]$. Let $I_{2}$ be that subarc of $\pi[A]$.

Define $r$ and $s$ to be the end points of $I_{2}$ belonging to $\left\langle p_{1}, q_{1}\right\rangle$ and $\left\langle p_{5}, q_{5}\right\rangle$ respectively. Let $D$ be the bounded complementary domain of $I_{2} U$ $\left\langle p_{1}, p_{5}\right\rangle \cup\left\langle p_{1}, r\right\rangle \cup\left\langle p_{5}, s\right\rangle$ in $R^{2}$.

Suppose $B$ is a component of $M \cap \pi^{-1}[D]$ that intersects $\bigcup\left\{L_{i}: 4 \leqslant i \leqslant 8\right\}$. Since $M$ is atriodic, it follows from Property 1 and Lemma 5 that $\mathrm{Cl} B$ is the image of a subcontinuum of $E$ (essential to $R(E)$ ) under an $\epsilon_{1}$-homeomorphism. Note that the chain $L-\left\{L_{1}, L_{11}\right\}$ follows $\mathrm{Cl} B$.

Define $\epsilon_{2}$ to be the minimum of $\epsilon_{1}$ and $\rho\left(M, \pi^{-1}\left[I_{1} \cup I_{2}\right]\right)$. By Lemma 7, there exists a positive number $\epsilon_{3}$ less than $\epsilon_{2}$ such that if $h$ is an $\epsilon_{3}$-homeomorphism of $M$ onto $M$, then $h[R(E)]$ has the following property:

Property 2. If $H$ is a continuum essential to $h[R(E)]$ with end sets $T$ and $V$ such that $\rho(T, V)<\epsilon_{3}$ and if there exists an element $U$ of the decomposition of $H$ such that $\rho(T, U)>\epsilon_{2}$, then each point of $R(E)$ is within $\epsilon_{2}$ of $H$. 
Let $K$ be the component of $\pi^{-1}\left[D \cap\left\langle p_{3}, q_{3}\right\rangle\right]$ whose closure meets both $\pi^{-1}\left(p_{3}\right)$ and $\pi^{-1}\left[I_{2}\right]$. Note that $K$ separates $L_{2} \cap M \cap \pi^{-1}[D]$ from $L_{10} \cap$ $M \cap \pi^{-1}[D]$ in $\pi^{-1}[D]$.

A point $p$ of $K$ and three $\epsilon_{3}$-homeomorphisms $f, h$, and $h_{1}$ of $M$ onto $M$ exist such that (1) $f=h_{1} h^{-1}$, (2) the closure of the $p$-component $C$ of $M \cap$ $\pi^{-1}[D]$ is essential to $h[R(E)]$, and (3) $R(E)$ separates $p$ from $f(p)$ in $\pi^{-1}[D]$. To see this let $x$ be a point of $\pi^{-1}\left(p_{3}\right) \cap \mathrm{Cl} K$. By Lemma $2, W=\{y \in M$ : an $\epsilon_{3} / 2$-homeomorphism of $M$ onto $M$ takes $x$ to $y$ \} is open in $M$. Let $p$ and $q$ be points of $K \cap(W-R(E))$ that belong to distinct inaccessible composants [10] of the indecomposable continuum $\mathrm{Cl} R(E)$ (Lemma 6).

Define $h$ and $h_{1}$ to be $\epsilon_{3} / 2$-homeomorphisms of $M$ onto $M$ such that $h(x)=$ $p$ and $h_{1}(x)=q$. Note that $f=h_{1} h^{-1}$ is an $\epsilon_{3}$-homeomorphism. Since $M$ is atriodic, it follows from Lemma 5 that $h$ maps a subcontinuum of $E$ that is essential to $R(E)$ onto $\mathrm{Cl} C$. By Property $1, R(E)$ intersects an arc in $K$ that is irreducible between $C$ and the $q$-component of $M \cap \pi^{-1}[D]$ and $R(E)$ separates these components in $\pi^{-1}[D]$. Hence $p, f, h$, and $h_{1}$ have the required properties.

Let $F$ be a continuum that is irreducible with respect to the following properties:

1. $F$ is an extension of $E$ in $M$ away from $k^{-1}(0)$.

2. $F$ separates $p$ from $f(p)$ in $\pi^{-1}[D]$.

The existence of such a continuum $F$ follows from Property 1 and the fact that $\mathrm{Cl} R(E)=\mathrm{Cl} R\left(k^{-1}(0), E\right)$ (Lemma 6 (proof)).

Let $d: F \rightarrow[0,1]$ be the quotient map associated with the decomposition of $F$. We assume $d^{-1}(0)=k^{-1}(0)$.

PROPERTY 3. No component of $F \cap \pi^{-1}[D]$ whose closure meets both $\pi^{-1}\left[\left\langle p_{1}, r\right\rangle\right]$ and $\pi^{-1}\left[\left\langle p_{5}, s\right\rangle\right]$ is contained in an element of the decomposition of $F$. To see this assume the contrary. Let $v$ be the first point of $[0,1]$ such that $d^{-1}(v)$ contains a component of $F \cap \pi^{-1}[D]$ whose closure meets $\pi^{-1}\left[\left\langle p_{1}, r\right\rangle\right]$ and $\pi^{-1}\left[\left\langle p_{5}, s\right\rangle\right]$. By Lemma 1 and Property $1, L$ follows a type $A^{\prime}$ continuum in $d^{-1}(v)$ that meets $L_{6} \cap \pi^{-1}[D]$. By Lemmas 2 and 3, a homeomorphism $g$ of $M$ onto $M$ and a point $u$ of $[0, v)$ exist such that $g d^{-1}(v)=$ $d^{-1}(u)$ and $d^{-1}(u)$ contains a component of $F \cap \pi^{-1}[D]$ whose closure meets $\pi^{-1}\left[\left\langle p_{1}, r\right\rangle\right]$ and $\pi^{-1}\left[\left\langle p_{5}, s\right\rangle\right]$. This contradicts the definition of $v$.

Let $I$ be the collection of intervals $[x, y]$ in $[0,1]$ such that $d^{-1}[[x, y]]$ is a continuum irreducible with respect to containing a component of $F \cap$ $\pi^{-1}[D]$ whose closure meets both $\pi^{-1}\left[\left\langle p_{1}, r\right\rangle\right]$ and $\pi^{-1}\left[\left\langle p_{5}, s\right\rangle\right]$. It follows from Property 3 that $I$ is finite.

Let $e$ be the point of $[0,1]$ such that $d^{-1}[[0, e]]=E$. Let $J=I \cup$ $\{[0, e]\}$. 
Define $\left\{x_{i}: 1 \leqslant i \leqslant 2 n\right\}$ to be the finite increasing sequence in $[0,1]$ such that St $J=\bigcup\left\{\left[x_{2 i-1}, x_{2 i}\right]: 1 \leqslant i \leqslant n\right\}$. Note that $x_{1}=0, x_{2}=e$, and $x_{2 n}=1$.

The interval $\left[x_{2 n-1}, x_{2 n}\right]$ has the following property:

Property 4. There exists a point $y_{n}$ of $K$ such that (1) the closure of the $y_{n}$-component $C_{n}$ of $M \cap \pi^{-1}[D]$ is essential to $h[R(E)]$, and (2) $d^{-1}\left[\left[x_{2 n-1}, x_{2 n}\right]\right]$ separates $y_{n}$ from $f\left(y_{n}\right)$ in $\pi^{-1}[D]$.

Note that $\left[x_{1}, x_{2}\right]$ does not have Property 4. Following Bing [2, p. 225], we now show that if $I=\left[x_{2 i-1}, x_{2 i}\right]$ has Property 4, then so does $J=$ $\left[x_{2 i-3}, x_{2 i-2}\right]$. The resulting contradiction will complete our proof.

We assume without loss of generality that $\pi^{-1}\left[I_{2}\right]$ is above $E$ and $\pi^{-1}\left[\left\langle p_{1}, r\right\rangle\right]$ is to the left of $\pi^{-1}\left[\left\langle p_{5}, s\right\rangle\right]$ in $R^{2}$.

Suppose $I$ has Property 4. For convenience we suppose $d^{-1}[J]$ is below $d^{-1}[\eta]$ and $f\left(y_{i}\right)$ is above $d^{-1}[\eta$. (Other cases are handled with similar arguments to that given in this case.) Then $d^{-1}[J]$ separates $y_{i}$ from $f\left(y_{i}\right)$ in $\pi^{-1}[D]$ and $J$ has Property 4 unless $y_{i}$ is above $d^{-1}[J]$, so we suppose $y_{i}$ is between $d^{-1}\left[\eta\right.$ and $d^{-1}[J]$.

Let $X$ and $Z$ be the end sets of $\mathrm{Cl} C_{i}$. The proof now breaks down into two cases.

Case 1. Suppose $d^{-1}\left(x_{2 i-1}\right)$ and $d^{-1}\left(x_{2 i-2}\right)$ intersect the same element of $\left\{\pi^{-1}\left[\left\langle p_{1}, r\right\rangle\right], \pi^{-1}\left[\left\langle p_{5}, s\right\rangle\right]\right\}$. We suppose $\pi^{-1}\left[\left\langle p_{1}, r\right\rangle\right]$ meets $Z$, $d^{-1}\left(x_{2 i-1}\right)$, and $d^{-1}\left(x_{2 i-2}\right)$.

Let $C_{i-1}$ be the first component of $M \cap \pi^{-1}[D]$ (whose closure intersects $\pi^{-1}\left[\left\langle p_{1}, r\right\rangle\right]$ and $\left.\pi^{-1}\left[\left\langle p_{5}, s\right\rangle\right]\right)$ that is met by extending $\mathrm{Cl} C_{i}$ away from $X$ in $M$. The existence of $C_{i-1}$ follows from the indecomposability of $\mathrm{Cl} R\left(X, \mathrm{Cl} C_{i}\right)$ (Lemma 6 (proof)) and an argument similar to the one for Property 3. Note that $\mathrm{Cl} C_{i-1}$ is essential to $h[R(E)]$. Let $L$ be the shortest extension of $\mathrm{Cl} C_{i}$ away from $X$ that contains $C_{i-1}$.

Let $y_{i-1}$ be a point of $K \cap C_{i-1}$. By considering the position of $d^{-1}\left[\left[x_{2 i-3}, x_{2 i}\right]\right]$, we see that $y_{i-1}$ is between $d^{-1}[\eta]$ and $d^{-1}[J]$. Let $N$ be the subcontinuum of $L$ essential to $h[R(E)]$ that has $y_{i}$ in one end set and $y_{i-1}$ in the other. Since $L-\left\{L_{1}, L_{11}\right\}$ follows $\mathrm{Cl} C_{i-1}$ and $\mathrm{Cl} C_{i}$, the continuum $N$ does not come within $\epsilon_{2}$ of $\pi^{-1}\left(p_{4}\right)$.

The point $y_{i-1}$ is below $C_{i}$ and $\rho\left(y_{i-1}, d^{-1}[\eta]\right) \geqslant \epsilon_{3}$; for otherwise, a subcontinuum of $L$ would violate Property 2. Note that $f\left(y_{i-1}\right)$ belongs to $L_{6} \cap \pi^{-1}[D]$.

We now show that $d^{-1}[J]$ separates $y_{i-1}$ from $f\left(y_{i-1}\right)$ in $\pi^{-1}[D]$. Consider the continuum $P$ in $R^{2}$ that is the union of $d^{-1}\left[\left[x_{2 i-3}, x_{2 i}\right]\right]$ and an arc segment $Q$ in $\pi^{-1}\left[D \cap\left\langle p_{4}, q_{4}\right\rangle\right]-d^{-1}\left[\left[x_{2 i-3}, x_{2 i}\right]\right]$ that has one end 
point in $d^{-1}[I]$ and the other in $d^{-1}[J]$. Since no point of $N$ is within $\epsilon_{2}$ of $Q$, the continuum $f[M]$ does not intersect $P$. Hence $f\left(y_{i-1}\right)$ is either above $d^{-1}[I]$ or below $d^{-1}[J]$. It is not above $d^{-1}[I]$ because $\rho\left(y_{i-1}, d^{-1}[I]\right) \geqslant \epsilon_{3}$ and $\rho\left(y_{i-1}, f\left(y_{i-1}\right)\right)<\epsilon_{3}$. Hence $J$ has Property 4.

Case 2. Suppose $d^{-1}\left(x_{2 i-1}\right)$ and $d^{-1}\left(x_{2 i-2}\right)$ intersect different elements of $\left\{\pi^{-1}\left[\left\langle p_{1}, r\right\rangle\right], \pi^{-1}\left[\left\langle p_{5}, s\right\rangle\right]\right\}$. We assume without loss of generality that $\pi^{-1}\left[\left\langle p_{5}, s\right\rangle\right]$ meets $Z, d^{-1}\left(x_{2 i-1}\right)$, and $d^{-1}\left(x_{2 i-3}\right)$. Define $C_{i-1}, L, y_{i-1}$, and $N$ as in Case 1.

If $y_{i-1}$ is above $d^{-1}[J]$, it is between $d^{-1}[I]$ and $d^{-1}[J]$, and $f\left(y_{i-1}\right)$ is above $d^{-1}\left[\Pi\right.$. Since both $y_{i}$ and $y_{i-1}$ are within $\epsilon_{3}$ of $d^{-1}[I]$, it follows as in Case 1 that a subcontinuum of $L$ violates Property 2.

Hence $y_{i-1}$ is below $d^{-1}[J]$ and $f\left(y_{i-1}\right)$ is above. Therefore $J$ has Property 4.

THEOREM 2. Every homogeneous plane continuum that does not separate the plane is hereditarily indecomposable.

Proof. Since every homogeneous nonseparating plane continuum is indecomposable [4] , [6], this theorem follows immediately from Theorem 1.

The following question, raised in [6], remains unanswered. Is every homogeneous nonseparating plane continuum a pseudo-arc [1], [11]?

\section{REFERENCES}

1. R. H. Bing, A homogeneous indecomposable plane continuum, Duke Math. J. 15 (1948), 729-742. MR 10, 261.

2. A simple closed curve is the only homogeneous bounded plane continuum that contains an arc, Canad. J. Math. 12 (1960), 209-230. MR 22 \#1869.

3. E. G. Effros, Transformation groups and C*-algebras, Ann. of Math. (2) 81 (1965), 38-55. MR 30 \#5175.

4. G. R. Gordh, Jr., On homogeneous hereditarily unicoherent continua, Proc. Amer. Math. Soc. 51 (1975), 198-202.

5. C. L. Hagopian, Homogeneous plane continua, Houston J. Math. 1 (1975), 35-41.

6. F. B. Jones, Certain homogeneous unicoherent indecomposable continua, Proc. Amer. Math. Soc. 2 (1951), 855-859. MR 13, 573.

7. On a certain type of homogeneous plane continuum, Proc. Amer. Math. Soc. 6 (1955), 735-740. MR 17, 180. Univ.,

8. Homogeneous plane continua, Proc. Auburn Topology Conf., Auburn

9. K. Kuratowski, Topology. Vol. 2, 3rd ed., Monografie Mat., Tom 21, PWN, Warsaw, 1961; English transl., Academic Press, New York; PWN, Warsaw, 1968. MR 41 \#4468.

10. S. Mazurkiewicz, Sur les points accessibles des continus indecomposables, Fund. Math. 14 (1929), 107-115.

11. E. E. Moise, $A$ note on the pseudo-arc, Trans. Amer. Math. Soc. 67 (1949), 57-58. MR 11, 382. 
12. R. L. Moore, Concerning upper semi-continuous collections of continua, Trans. Amer. Math. Soc. 27 (1925), 416-428.

13. - Foundations of point set theory, rev. ed., Amer. Math. Soc. Colloq. Publ., vol. 13, Amer. Math. Soc., Providence, R.I., 1962. MR 27 \#709.

14. E. S. Thomas, Jr., Monotone decompositions of irreducible continua, Rozprawy Mat. 50 (1966), 1-74. MR 33 \#4907.

DEPARTMENT OF MATHEMATICS, CALIFORNIA STATE UNIVERSITY, SACRAMENTO, CALIFORNIA 95819 